\title{
Tumor microenvironment contributes to Epstein-Barr virus anti-nuclear antigen-1 antibody production in nasopharyngeal carcinoma
}

\author{
PING AI ${ }^{1}$, ZHIPING LI $^{2}$, YONG JIANG ${ }^{3}, \mathrm{CHANGPING} \mathrm{SONG}^{4}, \mathrm{LIN} \mathrm{ZHANG}^{4}, \mathrm{HUAIZHONG} \mathrm{HU}^{4}$ and TAO WANG ${ }^{4}$ \\ Departments of ${ }^{1} \mathrm{Head}$ and Neck Oncology, and ${ }^{2}$ Abdominal Oncology, Cancer Center; ${ }^{3}$ Department of Pathology, West China \\ Hospital; ${ }^{4}$ Laboratory of Molecular and Translational Medicine, Department of Obstetrics and Gynecology, Key Laboratory \\ of Birth Defects and Related Diseases of Women and Children (Sichuan University), Ministry of Education, \\ West China Second University Hospital, Sichuan University, Chengdu, Sichuan 610041, P.R. China
}

Received November 11, 2015; Accepted April 24, 2017

DOI: $10.3892 /$ ol.2017.6461

\begin{abstract}
Nuclear antigen-1 (NA1) protein of Epstein-Barr virus (EBV) is expressed in EBV-infected cells in the microenvironment of cancer. Since immune cells infiltrate abundantly in nasopharyngeal carcinoma (NPC) tumor tissues, we hypothesized that the local tumor microenvironment may perform an important role in the production of antibodies directed at NA1. Furthermore, we hypothesized that anti-NA1 antibody originating in the local microenvironment could be secreted into the saliva of patients with NPC. In the present study, 20 healthy controls and 39 patients with NPC treated with intensity-modulated radiation therapy were recruited for the study. Saliva and serum samples were collected from the NPC patients, and nasopharyngeal tissue samples from the patients with NPC. The titers of anti-NA1 antibody [immunoglobulin A ( $\operatorname{IgA})$ ] were determined by ELISA. Expression of NA1, human leukocyte antigen-antigen D related (HLA-DR), cluster of differentiation (CD)80, CD86, CD3, CD4, CD19 and $\operatorname{IgA}$ was detected by immunohistochemical staining on paraffin-embedded nasopharyngeal tissue sections. Anti-NA1 antibodies were detected in the serum and saliva samples of the patients with NPC. In infiltrating cells, expression of HLA-DR, CD80, CD86, CD3, CD4, CD19 and IgA was detected, indicating that dendritic cells, $\mathrm{T}$ lymphocytes and B lymphocytes were all present in the local tumor tissues. Furthermore, expression of EBNA1 protein was detected on the membrane of the NPC tumor cells. Therefore, the NPC
\end{abstract}

Correspondence to: Dr Tao Wang, Laboratory of Molecular and Translational Medicine, Department of Obstetrics and Gynecology, Key Laboratory of Birth Defects and Related Diseases of Women and Children (Sichuan University), Ministry of Education, West China Second University Hospital, Sichuan University, 20 Ren Min Nan Lu, Chengdu, Sichuan 610041, P.R. China

E-mail: taowang@scu.edu.cn

Key words: tumor microenvironment, nasopharyngeal carcinoma, anti-nuclear antigen-1 antibody, nuclear antigen-1, immune cells tumor microenvironment has the potential to initiate a humoral response to EBNA1 by producing $\operatorname{Ig} \mathrm{A}$ antibodies.

\section{Introduction}

Nasopharyngeal carcinoma (NPC) is a distinctive type of head and neck malignant neoplasm that occurs globally, but is geographically distributed to a higher extent within South China and South East Asia. NPC is highly associated with Epstein-Barr virus (EBV) infection (1-5).

A number of different cell types can be infected by EBV, including B cells and epithelial cells $(6,7)$. Following the natural infection with EBV, the virus executes a distinct program of gene expression in the lytic or latent cycles to establish a persistent infection. Nuclear antigen-1 (NA1) protein is expressed during latent and lytic cycles, binding to a replication origin within the viral genome. It also mediates replication and partitioning of the episome during the division of the host cell, and is essential for the maintenance of the viral genome in latency (8-11). Since NA1 protein is expressed in EBV-infected cells, including cancer cells or cells in the cancer microenvironment, it may act as an antigen to induce immune responses if the microenvironment is suitable for the induction of humoral responses directed at NA1 (7).

To elicit humoral immune responses to induce the production of NA1 antibodies in the tumor local environment, the elements essential for a humoral response should all be present. These elements include antigens (NA1 in this case), antigen-presenting cells (APCs), T helper cells and $B$ cells (7). Since numerous immune cells infiltrate in the NPC tumor tissues, we hypothesized that the local tumor microenvironment may be adequate for the production of antibodies directed at NA1. Furthermore, since EBV is mainly transmitted via saliva, NA1 antibody originating in the local microenvironment may be secreted into the serum and saliva of patients with NPC (12). To confirm these hypotheses, serum samples and nasopharyngeal tissues were collected from patients with chronic inflammation with lymphoid hyperplasia and from patients with NPC, and antibodies directed at NA1 were detected. The results showed that NA1 antibodies were 
detected in the serum and saliva samples of patients with NPC, and that the local production of the antibodies could be completed in part in the local tumor microenvironment.

\section{Patients and methods}

Patients. The research protocol was approved by the Institutional Review Board of West China University Hospital, Sichuan University (Chengdu, China). All patients provided written informed consent and agreed to be involved in the present study. Between September 2011 and April 2012, 39 patients (34 male, 5 female; mean age, 46.9; age range 22-69) with pathologically confirmed NPC from West China University Hospital were enrolled in the present study. Each study patient was evaluated by flexible fiberoptic endoscopic examination, magnetic resonance imaging scans of the head and neck, chest X-ray, bone scan and ultrasound of the abdomen prior to treatment. X-ray computed tomography scans of the chest/abdomen were performed when clinically indicated. The histology of the tumor was evaluated according to the World Health Organization classification (13). Tumors were staged by Tumor-Node-Metastasis classification and clinical staging according to the American Joint Committee on Cancer 2009 cancer staging classification (13). NPC patient characteristics are presented in Table I.

A total of 20 healthy individuals (15 females and 5 males) were recruited for the present study (mean age, 24.8 years; range, 20-34 years).

Sample collection. Blood and saliva samples were collected from the healthy volunteers and from the patients with NPC prior to treatment. Vein blood $(1 \mathrm{ml})$ was collected into a sterile EDTA-containing vacutainer tube from each patient. Samples were then centrifuged at $400 \mathrm{x} \mathrm{g}$ and $4^{\circ} \mathrm{C}$ for $10 \mathrm{~min}$. The serum was collected, aliquoted and stored at $-80^{\circ} \mathrm{C}$ until use. At least $1 \mathrm{ml}$ saliva was collected using a sterile container. Samples were then centrifuged at $400 \mathrm{xg}$ and $4^{\circ} \mathrm{C}$ for $10 \mathrm{~min}$. The serum was collected, aliquoted and stored at $-80^{\circ} \mathrm{C}$ until use.

ELISA. Titers of antibodies directed at NA1 [immunoglobulin A $(\operatorname{Ig} \mathrm{A})]$ in the saliva and serum samples were determined in duplicate by ELISA using commercial kits (anti-EBNA1 ELISA kit; catalog no. 03-01-60402) purchased from Zhongshan Bio-Tech Co., Ltd. (Guangzhou, China). Samples with antibody levels higher than the detection limits were diluted and measured again. In the Laboratory of Molecular and Translational Medicine, West China Second University Hospital, Sichuan University, Chengdu, China), the intra-assay and inter-assay coefficients of variation were $\leq 10 \%$ for all assays. The experiments were conducted by following the manufacturer's suggested procedures. Two wells of blank controls (sample dilution buffer), two negative controls and two positive controls (as provided in the kit) were included on each plate. Briefly, diluted saliva and serum samples were added to each well (100 $\mu \mathrm{l}$ in each well) of a 96-well plate. Following incubation at room temperature (RT) for $2 \mathrm{~h}$ on a microplate agitator rotating at $25 \mathrm{~g}$, the plate was then washed with $1 \mathrm{X}$ PBS wash buffer with $0.05 \%$ Tween-20. Horseradish peroxidase (HRP)-conjugated anti-human IgA antibody from

Table I. Clinical characteristics of patients with NPC in the present study.

Characteristics

Total, $\mathrm{n}$

Gender, $\mathrm{n}$

Male

Female

Mean age (range), years

Histology, $\mathrm{n}$

Squamous cell carcinoma, poorly

differentiated, non-keratinized

Carcinoma, undifferentiated,

non-keratinized

Tumor stage, $\mathrm{n}$

I

II

III

IV

Depth of tumor invasion, $\mathrm{n}$

$\mathrm{T} 1$

T2

T3

T4

$\mathrm{N}$ stage, $\mathrm{n}$

N0

N1

$\mathrm{N} 2$

N3

M stage, $n$

M0

M1

Metastases, $\mathrm{n}$

Male

7

Female

1

Metastasis region, $\mathrm{n}$

Lung

Bone

Multiple regions (liver, lung, 2

39

1 2 8 11 18

16

14

7 bone, lymph node)

NPC, nasopharyngeal carcinoma.

the kit $(100 \mu \mathrm{l})$ was added to each well. Following incubation at RT for $20 \mathrm{~min}$, the plate was then washed with 1X PBS wash buffer. Color development was conducted by addition of $100 \mu \mathrm{l}$ tetramethylbenzidine substrate solution and incubation at RT for $10 \mathrm{~min}$. Once the reaction was stopped, optical absorbance (OA) was immediately read on a spectrophotometer (Infinite M200; Tecan Trading AG, Männedorf, Switzerland) using $450 \mathrm{~nm}$ as the primary wavelength and $630 \mathrm{~nm}$ as the secondary wavelength. OA value at a wavelength of $450 / 630 \mathrm{~nm}$ for each test was determined. 


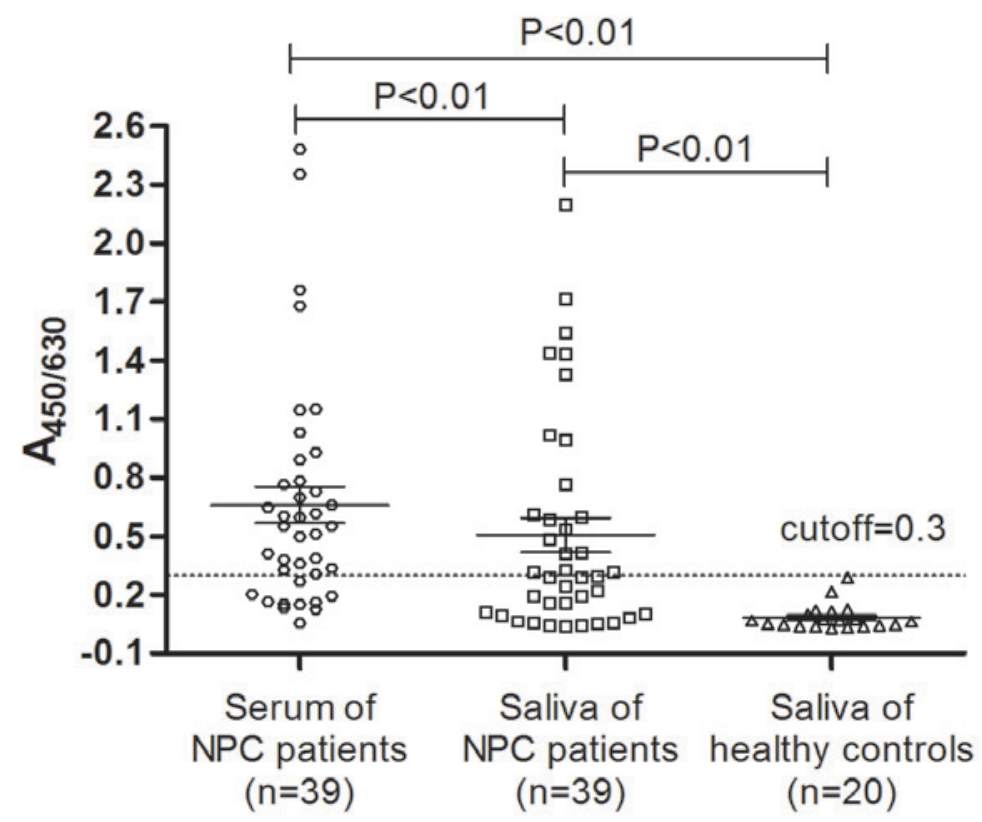

Figure 1. Optical absorbance value at wavelength $450 / 630 \mathrm{~nm}$ for anti-nuclear angiten-1 antibody in the saliva and serum samples of patients with NPC prior to anticancer treatment and in the saliva of healthy controls. NPC, nasopharyngeal carcinoma; A, absorbance.

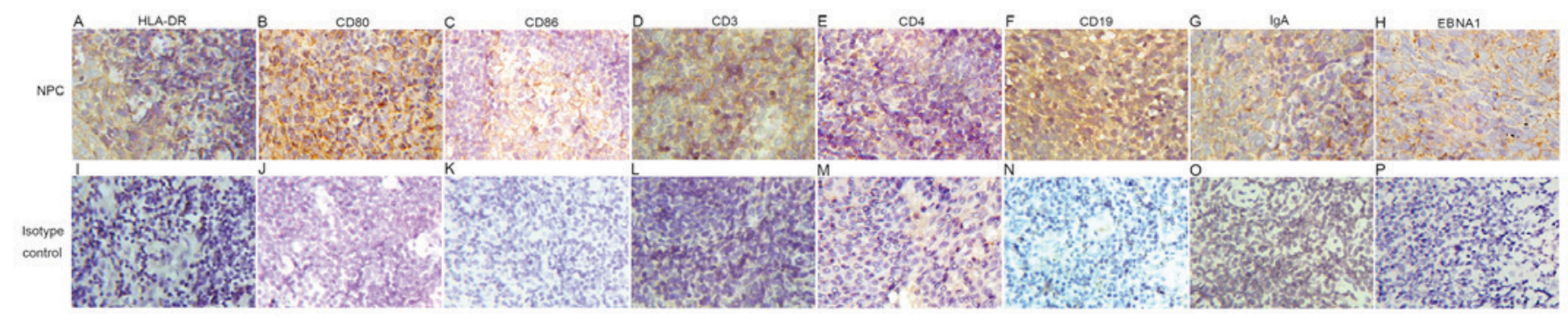

Figure 2. Expression of (A) HLA-DR, (B) CD80, (C) CD86, (D) CD3, (E) CD4, (F) CD19, (G) IgA and (H) EBNA1 was detected in the nasopharyngeal tissue by immunohistochemistry. When the primary antibody was replaced with the isotype control of (I) goat serum, (J) mouse IgG1, (K) rabbit IgG, (L) mouse (M) IgG2a or (N-P) $\kappa$, the positive signals were not observed (original magnification, x4,000). HLA-DR, human leukocyte antigen-antigen D related; CD, cluster of differentiation; IgA, immunoglobulin; EBNA1, Epstein-Barr virus nuclear antigen-1.

Immunohistochemistry. The immunohistochemical (IHC) staining was performed on paraffin-embedded nasopharyngeal tissue sections from 39 NPC patients. As shown in goat anti-human CD80 polyclonal antibody (1:100; IgG; catalog no. AF140; R\&D Systems, Inc., Minneapolis, MN, USA), goat anti-human CD86 polyclonal antibody (1:100; IgG; catalog no. AF-141-NA; R\&D Systems, Inc.), mouse anti-human CD4 monoclonal antibody (1:100; IgG1; catalog no. MAB379; R\&D Systems, Inc.), rabbit anti-human CD3 polyclonal antibody (1:100; IgG; catalog no. ab5690; Abcam, Cambridge, UK), mouse anti-human CD19 monoclonal antibody (1:100; IgG2a, $\kappa$; catalog no. ab31947; Abcam), mouse anti-human human leukocyte antigen-antigen D related (1:100; HLA-DR) monoclonal antibody (IgG1; catalog no. ab20181; Abcam), mouse anti-human IgA monoclonal antibody (1:300; IgG1; catalog no. GTX17839; GeneTex, Inc., Irvine, CA, USA) or mouse anti-human EBNA1 antibody (1:300; IgG1; catalog no. GTX40777; GeneTex) were used as the primary antibodies for IHC staining. A negative control was set up using isotype control of goat serum (Zhongshan Golden Bridge Biotechnology Co., Ltd., Beijing, China), mouse IgG1, rabbit IgG, mouse IgG2a or mouse $\kappa$ as the substitute for the primary antibody, respectively, according to the source of the primary antibody. All antibodies were diluted in PBS containing $0.1 \%$ bovine serum albumin (Roche Applied Science, Rotkreuz, Switzerland) and $0.01 \%$ sodium azide. Briefly, 4- $\mu \mathrm{m}$ tissue sections were deparaffinized for $15 \mathrm{~min}$ in xylene, hydrated in gradient ethyl alcohol of 100, 95, 85, 80 and $75 \%$, and then incubated with $3 \%$ hydrogen peroxidase for $10 \mathrm{~min}$ at room temperature to block endogenous peroxidase. Following antigen retrieval in $0.1 \mathrm{~mol} / \mathrm{l}$ citrate buffer $(\mathrm{pH}$ 6.0) for $15 \mathrm{~min}$ at $95^{\circ} \mathrm{C}$ in a pressure cooker, sections were cooled to RT and washed three times with PBS, and then incubated with the aforementioned diluted primary antibodies at $4^{\circ} \mathrm{C}$ overnight. Subsequently, sections were rewarmed to RT for $30 \mathrm{~min}$ and washed three times with PBS and then incubated with HRP-conjugated goat anti-rabbit/mouse secondary antibody (1:100; catalog no. ab6720; Dako; Agilent Technologies, Inc., Santa Clara, CA, USA) for $40 \mathrm{~min}$ at $37^{\circ} \mathrm{C}$. Diaminobenzidine was used at room temperature for 3 min for color development. Finally, the sections were counterstained with Mayer's hematoxylin and mounted in Permount (ZSGB-BIO, Beijing, China). Subsequently, light microscopy observation 
(magnification, $\mathrm{x} 4,000$; 5 fields of view) was performed. The reactivity of cell type and location in the immunostained tissues were determined.

Statistical analysis. OA values at wavelength 450/630 nm of NA1 IgA in saliva and serum samples are expressed as the mean value \pm standard deviation. The statistical significance of the findings was assessed by one-way analysis of variance using Prism 5 from GraphPad Software (GraphPad Software, Inc., La Jolla, CA, USA). P<0.05 was considered to indicate a statistically significant difference.

\section{Results}

Anti-NA1 in the serum and saliva. As shown in Fig. 1, antibody titers directed at NA1 were elevated in the saliva and serum of the majority of NPC patients, and anti-NA1 antibody titer in the serum was significantly higher than that of in the saliva of NPC patients $(\mathrm{P}<0.01)$. The cut-off value was defined as $\mathrm{A}_{450 / 630}$ of 0.3. Compared with that of the saliva of the healthy control, the anti-NA1 antibody titer in the saliva of the NPC patients was significantly higher $(\mathrm{P}<0.01)$. Anti-NA1 antibody titer was determined to be negative in the saliva of the 20 healthy volunteers; however, $43.6 \%$ (17/39) of the patients with NPC were positive for the antibody. Furthermore, $74.4 \%$ (29/39) of the patients with NPC were positive for anti-NA1 antibodies in the sera.

Expression of HLA-DR, CD80, CD86, CD3, CD4, CD19, $\operatorname{IgA}$, EBNA1 in the nasopharyngeal tissue. As shown in and Fig. 2, HLA-DR, CD80, CD86, CD3, CD4, CD19, IgA and EBNA1 antibodies were used as the primary antibodies for IHC staining. A negative control was set up using an isotype control of goat serum, mouse IgG1, rabbit IgG, mouse IgG2a or mouse $\kappa$ as the substitute for each primary antibody, respectively, according to the source of the primary antibody.

When the control antibodies were used, no positive signals were noted on tissue sections from patients with NPC. In infiltrating cells, the expression of HLA-DR, CD80 and CD86 was detected at the membrane of dendritic cells, the expression of CD3 and CD4 was detected at the membrane of T lymphocytes, the expression of CD19, $\operatorname{IgA}$ was detected at the membrane of B lymphocytes and the expression of EBNA1 protein was highly expressed at the membrane of the tumor cells in NPC tissue specimens (Fig. 2).

\section{Discussion}

The level of serum antibodies directed at the EBNA1 antigen during latent and lytic cycles is elevated in patients with NPC and has been used as a serological marker for NPC diagnosis $(14,15)$. Combined detection of anti-NA1, anti-early antigen (EA), anti-viral capsid antigen and anti-replication and transcription activator increases the sensitivity and has been considered as a useful serological method for NPC screening and diagnosis (16-20). Salivary IgA antibody titers were previously reported to be increased in response to EA, gp340 or NA1-p107 in healthy individuals compared with patients with NPC. However, these salivary parameters were poor in sensitivity and specificity for NPC screening and diagnosis $(12,21,22)$. To the best of our knowledge, the present study is the first to report that anti-NA1 antibodies are elevated in the saliva and serum of patients with NPC. Although the detection of anti-NA1 antibody in saliva is non-invasive, quick and convenient, it has a limited value for clinical use due to its low sensitivity $(12,21,22)$.

The present study investigated whether anti-NA1 antibodies could be induced in the local tumor environment. EBNA1 is the only viral protein consistently expressed during all forms of latency and in all EBV-associated malignancies $(9,10)$. It is potentially a universal target for immune recognition of EBV-infected normal or malignant cells (10). The series of essential components for a humoral immune response, including NA1-expressed cells, APCs (HLA-DR+, CD80+ and CD86+), T helper cells (CD3+ and CD4+) and B cells (CD19+ and IgA+), were all detected by IHC staining in the local tumor environment. This indicated that anti-NA1 antibodies may be induced in the local tumor environment. The current study provides a novel insight into the use of EBNA1 antibodies and indicates that the tumor microenvironment contributes to the production of EBNA1 antibodies. Further study into the role of EBNA1 antibodies in the pathogenesis of EBV-related inflammation and NPC would, however, be useful to expand upon the present data.

\section{Acknowledgements}

The present study was supported by the Special Research Foundation of Doctoral Priority to the Development of Field Project (grant no. 20110181130013).

\section{References}

1. Chen JN, Ding YG, Feng ZY, Li HG, He D, Du H, Wu B and Shao CK: Association of distinctive Epstein-Barr virus variants with gastric carcinoma in Guangzhou, southern China. J Med Virol 82: 658-667, 2010

2. Deng Z, Hasegawa M, Matayoshi S, Kiyuna A, Yamashita Y, Maeda $\mathrm{H}$ and Suzuki M: Prevalence and clinical features of human papillomavirus in head and neck squamous cell carcinoma in Okinawa, southern Japan. Eur Arch Otorhinolaryngol 268: 1625-1631, 2011.

3. Garandawa HI, Ahmad BM and Nggada HA: Nasopharyngeal cancer in North-Eastern Nigeria: Clinical trends. Niger J Clin Pract 12: 379-382, 2009.

4. Popat SR, Liavaag PG, Morton R, McIvor N, Irish JC and Freeman JL: Epstein Barr virus genome in nasopharyngeal carcinomas from New Zealand. Head Neck 22: 505-508, 2000.

5. Rathaur RG, Chitale AR and Banerjee K: Epstein-Barr virus in nasopharyngeal carcinoma in Indian patients. Indian J Cancer 36: 80-90, 1999.

6. Valencia SM and Hutt-Fletcher LM: Important but differential roles for actin in trafficking of Epstein-Barr virus in B cells and epithelial cells. J Virol 86: 2-10, 2012.

7. Shannon-Lowe C, Adland E, Bell AI, Delecluse HJ, Rickinson AB and Rowe M: Features distinguishing Epstein-Barr virus infections of epithelial cells and B cells: Viral genome expression, genome maintenance and genome amplification. J Virol 83: 7749-7760, 2009

8. Gaur N, Gandhi J, Robertson ES, Verma SC and Kaul R: Epstein-Barr virus latent antigens EBNA3C and EBNA1 modulate epithelial to mesenchymal transition of cancer cells associated with tumor metastasis. Tumour Biol 36: 3051-3060, 2015.

9. Kang MS and Kieff E: Epstein-Barr virus latent genes. Exp Mol Med 47: e131, 2015.

10. Palser AL, Grayson NE, White RE, Corton C, Correia S, Ba Abdullah MM, Watson SJ, Cotten M, Arrand JR, Murray PG, et al: Genome diversity of Epstein-Barr virus from multiple tumor types and normal infection. J Virol 89: 5222-5237, 2015. 
11. Tierney RJ, Shannon-Lowe CD, Fitzsimmons L, Bell AI and Rowe M: Unexpected patterns of Epstein-Barr virus transcription revealed by a high throughput PCR array for absolute quantification of viral mRNA. Virology 474: 117-130, 2015.

12. Foong YT, Cheng HM, Sam CK, Dillner J, Hinderer W and Prasad U: Serum and salivary IgA antibodies against a defined epitope of the Epstein-Barr virus nuclear antigen (EBNA) are elevated in nasopharyngeal carcinoma. Int $\mathbf{J}$ Cancer 45 : 1061-1064, 1990.

13. Finger PT; 7th Edition, AJCC-UICC Ophthalmic Oncology Task Force: The 7th edition AJCC staging system for eye cancer: An international language for ophthalmic oncology. Arch Pathol Lab Med 133: 1197-1198, 2009.

14. Cheng HM, Foong YT, Sam CK, Prasad U and Dillner J: Epstein-Barr virus nuclear antigen 1 linear epitopes that are reactive with immunoglobulin $\mathrm{A}(\mathrm{Ig} \mathrm{A})$ or $\mathrm{IgG}$ in sera from nasopharyngeal carcinoma patients or from healthy donors. J Clin Microbiol 29: 2180-2186, 1991.

15. Tedeschi R, Pin E, Martorelli D, Bidoli E, Marus A, Pratesi C, Bortolin MT, Zanussi S, Vaccher E, Dolcetti R and De Paoli P: Serum antibody response to lytic and latent Epstein-Barr virus antigens in undifferentiated nasopharyngeal carcinoma patients from an area of nonendemicity. Clin Vaccine Immunol 14: 435-441, 2007.

16. Tang JW, Rohwäder E, Chu IM, Tsang RK, Steinhagen K, Yeung AC, To KF and Chan PK: Evaluation of Epstein-Barr virus antigen-based immunoassays for serological diagnosis of nasopharyngeal carcinoma. J Clin Virol 40: 284-288, 2007.
17. Paramita DK, Fachiroh J, Haryana SM and Middeldorp JM: Evaluation of commercial EBV RecombLine assay for diagnosis of nasopharyngeal carcinoma. J Clin Virol 42: 343-352, 2008.

18. Karray H, Ayadi W, Fki L, Hammami A, Daoud J, Drira MM, Frikha M, Jlidi R and Middeldorp JM: Comparison of three different serological techniques for primary diagnosis and monitoring of nasopharyngeal carcinoma in two age groups from Tunisia. J Med Virol 75: 593-602, 2005.

19. Cho WC: Nasopharyngeal carcinoma: Molecular biomarker discovery and progress. Mol Cancer 6: 1, 2007.

20. Ai P, Wang T, Zhang H, Wang Y, Song C, Zhang L, Li Z and $\mathrm{Hu} \mathrm{H}$ : Determination of antibodies directed at EBV proteins expressed in both latent and lytic cycles in nasopharyngeal carcinoma. Oral Oncol 49: 326-331, 2013.

21. Yao QY, Rowe M, Morgan AJ, Sam CK, Prasad U, Dang H, Zeng Y and Rickinson AB: Salivary and serum IgA antibodies to the Epstein-Barr virus glycoprotein gp340: incidence and potential for virus neutralization. Int J Cancer 48: 45-50, 1991.

22. Nadala EC, Tan TM, Wong HM and Ting RC: ELISA for the detection of serum and saliva IgA against the BMRFI gene product of Epstein-Barr virus. J Med Virol 50: 93-96, 1996. 